\title{
Edle Platte mit verborgener Technik
}

Manch einer hat "es" vermutlich in der Bibliothek schon einmal gesehen: Das knapp $40 \times 30 \mathrm{~cm}$ große "Ding" mit edler schwarzer Acrylglasoberfläche. Dieser Gegenstand kann gut sichtbar auf dem Schreibtisch liegen; bei den meisten entsprechend ausgestatteten Arbeitsplätzen ist dieses Gerät jedoch etwas versteckt unter der Schreibtischplatte befestigt. Diese Blackbox soll nun im Folgenden etwas unter die Lupe genommen werden. Es handelt sich dabei um eine flache, abgeschirmte Antenne mit integrierter Leseelektronik und ist ein notwendiges Gerät, um RFIDgestützte Geschäftsgänge umsetzen zu können. RFID steht für "radio-frequency identification"; ein RFID-System dient also der "Identifizierung mit Hilfe elektromagnetischer Wellen" und besteht im Wesentlichen aus drei Komponenten: Den RFIDTranspondern, der RFID-Antenne mit Leseelektronik und der Software, die diese Technik integrieren kann. RFID-Technik soll durch eine automatisierbare Identifikation von Medien die Geschäftsgänge beschleunigen.

Der RFID-Transponder besteht aus einer Antenne und einem kleinen Chip, der unter anderem einen beschreibbaren Datenspeicher beinhaltet. Diese elektronischen Teile werden auf ein flexibles

\section{Württembergische} Landesbibliothek Stuttgart

\section{WL82019}

Abb. 1: RFID-Etikett, fotografiert als Durchlichtaufnahme

klebbares Etikett "gepackt" (Abb. 1). Üblich sind dabei die Etikettenformate $9 \times 5 \mathrm{~cm}$ und $5 \times 5 \mathrm{~cm}$, wobei größere Etiketten besser detektiert werden können. Für die RFID-Transponder sind auch die
Bezeichnungen RFID-Tag oder RFID-Etikett üblich. Es ist ein günstiges Massenprodukt, das in die Medien eingeklebt werden kann. Im Datenspeicher des Tags werden nach dem im bibliothekarischen Umfeld üblichen dänischen Datenmodell nur Exemplar- und Statusdaten (Mediennummer, Sigel, Sicherungszustand, usw.) abgelegt, jedoch keine personenbezogenen oder personenbeziehbaren Daten.

Es handelt sich dabei um ein passives elektronisches Element. Passiv meint in diesem Zusammenhang, dass es auf dem Tag keine eigene Energieversorgung gibt. Es stellt sich nun die Frage, wie mit einem passiven elektronischen Element Kontakt aufgenommen werden kann, um mit den dort abgelegten Daten arbeiten zu können. Hier kommt nun die RFID-Antenne (Abb. 2) ins Spiel. Eine Antenne stellt eine Verbindung zwischen einem leitungsgebundenen System und dem freien Raum her und kann elektromagnetische Signale absenden und empfangen. Diese elektromagnetische Welle ist ein Energiefeld. Befindet sich nun in der Nähe der Antenne ein RFID-Transponder, so wird dessen Antenne vom elektromagnetischen Feld (EM-Feld) durchdrungen und es wird eine Spannung induziert, die ausreicht, um für die Aufenthaltszeitdauer im EM-Feld den Chip zu aktivieren. Damit das funktioniert, muss die Antenne mit einer Frequenz senden, die der Resonanzfrequenz des Transponders (Schwingkreis!) entspricht. Bei Systemen, die in Bibliotheken zur Anwendung kommen, wird üblicherweise mit der Frequenz 13,56 MHz gearbeitet. In diesem Resonanzzustand kann ein RFID-Etikett seine Daten auf den Funkträger modulieren und ein Datenaustausch ist möglich. Etiketten können bis zu einer Entfernung von 35-60 cm ausgelesen werden - abhängig von der Sendeleistung der Antenne. Da es sich um eine Funktechnik handelt, ist ein Sichtkontakt wie beim Barcode nicht nötig.

Die oben erwähnte Blackbox (Abb. 2) beinhaltet neben der Antenne auch noch eine Leseelektronik. Diese hat die Aufgabe die Nutzdaten zu 
extrahieren, da der Datenstrom zur sicheren Datenübertragung üblicherweise um Prüfziffern, Fehlermanagementdaten, Formatinformationen usw. angereichert ist. Angeschlossen wird die Antenne mit Leseeinheit über die USB-Schnittstelle an einen PC, optional ist auch eine LAN-Anbindung möglich. Durch den Leistungsbedarf der Antenne ist in der Regel eine separate Stromversorgung für den Antennenbetrieb notwendig.

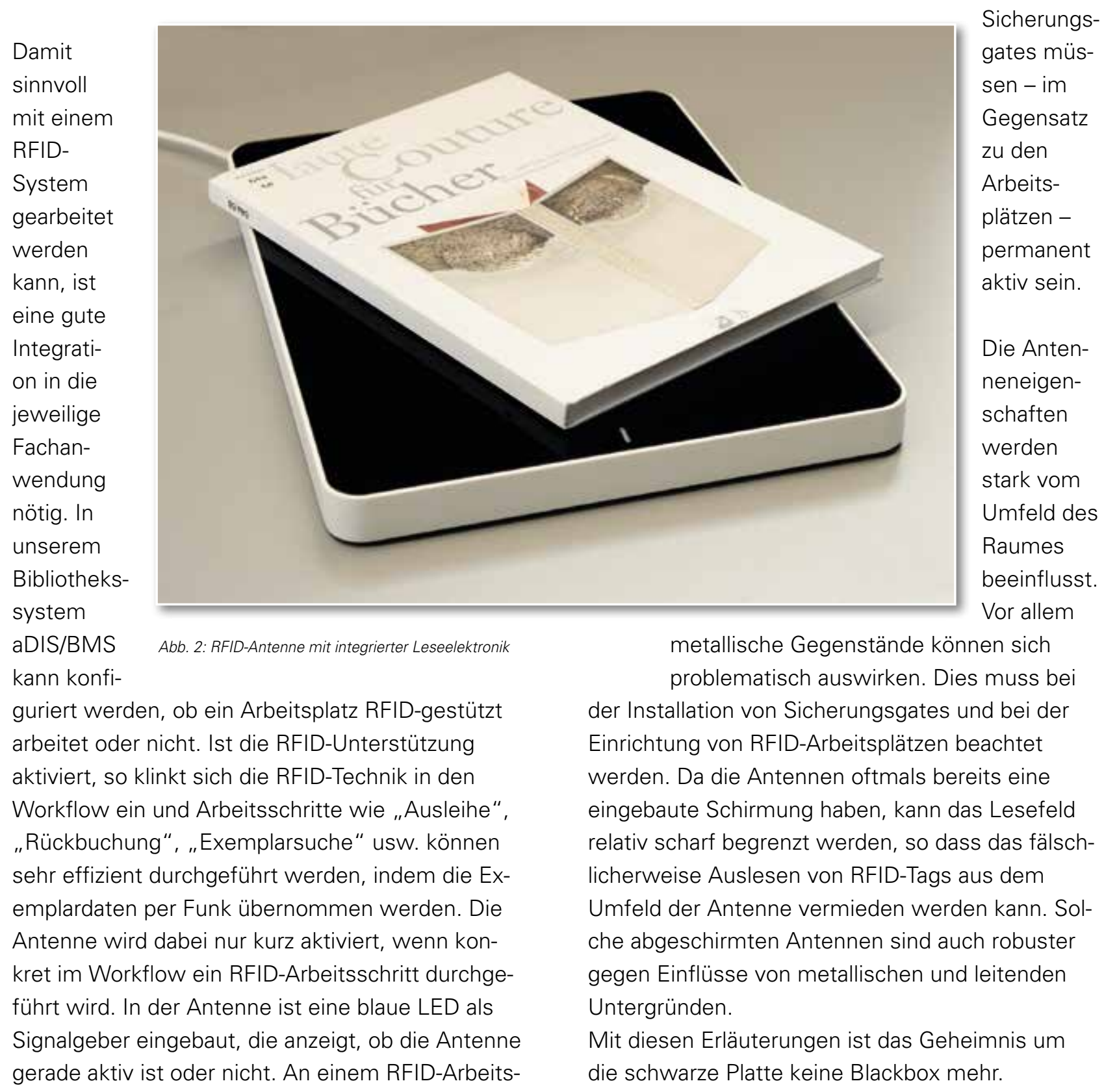

wird auf dem RFID-Transponder ein sogenanntes AFI-Byte entsprechend gesetzt. Wird nun ein Buch mit gesichertem AFI-Byte durch ein RFIDSicherungsgate getragen, so wird dies erkannt und Alarm ausgelöst. Für diese Alarmierung ist keine Verknüpfung zum Bibliothekssystem notwendig, so dass der Alarm sehr schnell ausgelöst werden kann und auch funktioniert, wenn das Bibliothekssystem offline sein sollte. Die Antennen in den

Sicherungs- 